\title{
Desempenho do Distrito de Irrigação Senador Nilo Coelho ${ }^{1}$
}

\author{
Gustavo H. F. de Souza ${ }^{2}$, Ricardo A. L. Brito ${ }^{3}$, José Dantas Neto ${ }^{4}$, José M. Soares ${ }^{5} \&$ Tarcizio Nascimento $^{6}$ \\ 1 Parte da Dissertação de Mestrado apresentada pelo primeiro autor à UFPB, financiada pela SRH/IICA e Gestão \\ Operacional da FAPED, através do projeto RPIP, Brasil \\ 2 R. João F. da Mota, 309, Catolé, CEP 58104-593, Campina Grande, PB, E-mail: gustavohfs@bol.com.br (Foto) \\ ${ }^{3}$ CNPMS/EMBRAPA. CEP 35701-970, Sete Lagoas, MG. E-mail: rbrito@cnpms.embrapa.br \\ ${ }^{4}$ DEAg/UFPB. CEP 58109-970, Campinas Grande, PB. E-mail: zedantas@deag.ufpb.br \\ ${ }^{5}$ CPATSA/EMBRAPA. CEP 56300-970, Petrolina, PE. E-mail: monteiro@cpatsa.embrapa.br \\ 6 CPATSA/EMBRAPA. E-mail: tarcizio@cpatsa.embrapa.br
}

Protocolo $056-29 / 05 / 2000$

\begin{abstract}
Resumo: O presente trabalho teve como objetivo descrever e avaliar, de maneira global e quantitativa, o uso de indicadores de desempenho destinados a orientar intervenções para o aprimoramento do desempenho em projetos de irrigação. Com a implementação do programa de pesquisa sobre avaliação do desempenho da irrigação no Brasil (Projeto RPIP-Brasil) uma das áreas-piloto selecionadas foi o Distrito de Irrigação Senador Nilo Coelho. Vários indicadores relacionados à área irrigada, ao requerimento e fornecimento de água, à energia e a tarifa de água, foram quantificados e analisados, apresentando um perfil do desempenho do perímetro irrigado, além de apresentarem resultados promissores ao longo dos anos verificando-se, também, uma interdependência entre eles.
\end{abstract}

Palavras-chave: irrigação, projetos, desempenho

\section{Performance of the Irrigated District Senador Nilo Coelho - Brazil}

\begin{abstract}
The present work had the objective of describing and evaluating, with a global and quantitative approach, the use of performance indicators aimed at guiding interventions for the improvement of performance in irrigation schemes. With the implementation of the research program to evaluate irrigation performance in Brazil (project RPIP-Brazil), one of the selected pilot areas was the Irrigation District Senator Nilo Coelho. Various indicators, related to irrigated area, water requirements, water supply, energy and water tariff, were quantified and analysed, showing a profile of the irrigation scheme performance. The indicators utilized presented promising results along the years of monitoring and showed an inter-dependent relationship among them.
\end{abstract}

Key words: irrigation, schemes, performance

\section{INTRODUÇÃO}

O Distrito de Irrigação Senador Nilo Coelho - DISNC, está localizado na margem esquerda do Rio São Francisco, no Nordeste brasileiro, e se estende desde a Barragem de Sobradinho, no município de Casa Nova, BA, até o município de Petrolina, PE (latitude $09^{\circ} 09^{\prime} \mathrm{S}$, longitude $40^{\circ} 22^{\prime} \mathrm{W}$ ) que tem cerca de $80 \%$ da área do Perímetro. O projeto tem uma área de 15.000 ha em operação, com 1.457 lotes para área de colonização, que respondem por $60 \%$ da área irrigável, e 132 lotes para a área empresarial, com $40 \%$ desta mesma área, dividida em 11 núcleos. A captação de água é feita no dique "B" da barragem de Sobradinho, BA, de propriedade da Companhia Hidroelétrica do São Francisco - CHESF.
Este trabalho baseia-se em uma proposta apresentada durante a Reunião do Conselho Executivo da Comissão Internacional de Irrigação e Drenagem - ICID, por ocasião do XVI Congresso Internacional de Irrigação e Drenagem realizado no Cairo, Egito, em setembro de 1996, com base metodológica no "Research Program on Irrigation Performance" (Programa de Pesquisa sobre Desempenho de Irrigação - Projeto RPIP) o qual visa à definição de um conjunto básico de indicadores destinados a orientar intervenções para o aprimoramento de projetos de irrigação.

A avaliação do desempenho deve ser feita por várias razões, incluindo-se: a melhoria operacional do sistema; a avaliação do progresso em relação às metas estratégicas, como parte do manejo integrado; a avaliação da situação geral do projeto; a 
comparação do desempenho de um projeto com outros, ou do mesmo projeto, ao longo do tempo. Os tipos de indicadores a serem escolhidos dependem do objetivo da atividade de avaliação (Molden et al., 1998).

De acordo com Brito (1986) diversos fatores contribuem para o desempenho dos perímetros irrigados, sejam eles técnicos, econômicos ou sociais. Um dos aspectos primordiais na análise desses fatores é a possibilidade de monitorá-los, o que significa transformá-los, de algum modo, em parâmetros mensuráveis. O nível desse monitoramento deverá estar situado entre o "desejável" e o "viável", considerando-se, para isso, o padrão da avaliação que se pretende proceder e os custos operacionais necessários à medição dos indicadores.

O monitoramento é um instrumento gerencial de suma importância para a tomada de decisões, uma vez que permite avaliar-se o desempenho das diversas atividades, acompanhar a evolução deste desempenho ao longo do tempo, adotar medidas corretivas necessárias e, no caso específico das atividades de operação e manutenção, comparar os indicadores com perímetros irrigados similares (Effertz et al., 1993).

Muitos indicadores de desempenho são descritos na literatura e diversas estruturas conceituais para avaliar o desempenho da irrigação, têm sido propostas; todavia, é difícil aplicá-los, atualmente, para uma análise comparativa entre projetos de irrigação, pois não existe uma concordância entre os especialistas sobre quais indicadores a serem utilizados. Ademais, há uma carência de dados disponíveis sobre o desempenho dos projetos de irrigação que podem ser usados para a análise comparativa (Merrey, 1996).

Segundo Bos (1997) existe cerca de 40 indicadores de desempenho multidisciplinares atualmente utilizados no Projeto RPIP, os quais cobrem a distribuição e o uso eficiente da água, manutenção, sustentabilidade da irrigação, aspectos ambientais, socioeconômicos e de manejo. De acordo com o autor, ainda estão em fase de teste a nível de campo, para posteriormente serem recomendados para o uso na avaliação do desempenho de perímetros irrigados.

Portanto, os indicadores demonstram, ao longo do tempo, resultados e modificações encontrados no projeto analisado, de forma a poder sinalizar o alcance das metas previstas; assim, o acompanhamento contínuo por meio de indicadores de desempenho de um projeto torna-se uma atividade obrigatória na análise dos fatores que conduzem à obtenção das metas pretendidas.

Embora existam sugestões de cerca de 40 indicadores, é importante ter-se em mente que a avaliação visa ser uma ferramenta para os gerentes de projeto e, portanto, o número de indicadores não pode ser muito extenso, pois inviabilizaria, do ponto de vista prático, a operacionalidade da avaliação.

A partir do conjunto básico de indicadores definidos pelo grupo de trabalho do Projeto RPIP e em função da disponibilidade dos dados já existentes, selecionaram-se alguns deles para avaliação do desempenho do DISNC. A seguir, são descritos os indicadores de desempenho, juntamente com suas relações e definições dos parâmetros utilizados.

\section{Sustentabilidade da área irrigada}

A definição da área de cada lote num perímetro irrigado, é feita com base em critérios preestabelecidos e todas as previsões adotadas no planejamento do projeto consideram a utilização de toda a área implantada. Conseqüentemente, a ocupação ou operação parcial da área pelo usuário significa ociosidade na capacidade produtiva, seja por dificuldades encontradas ou por falta de motivação suficiente. Qualquer que seja a causa, ela prejudica o bom desempenho do projeto e precisa ser medida e analisada (Brito, 1986).

Este indicador é obtido da relação entre a área irrigada (ha) e a área irrigável (ha) descrevendo a ocupação da área ao longo do tempo, e definida pela seguinte relação:

$$
\text { Sustentabilidade da área irrigada }=\frac{\text { Área irrigada }}{\text { Área irrigável }}
$$

A área irrigável representa o potencial de área que pode, efetivamente, ser cultivada com irrigação.

\section{Indicadores do balanço hídrico}

Razão global de consumo: O volume de água fornecido ao projeto é determinado como função do uso consuntivo das culturas; em outras palavras, é a água fornecida que poderia adequadamente alcançar as necessidades das culturas no projeto. Como a precipitação efetiva $(\mathrm{Pe})$ pode suprir as necessidades da cultura, em parte ou completamente, é preciso descontá-la da evapotranspiração potencial (ETp) fornecendo, deste modo, uma estimativa mais realista do requerimento de água na área irrigada (Brito et al.,1998). A razão global de consumo é então definida como:

$$
\text { Razão Global de Consumo }=\frac{\text { ETp }- \text { Pe }}{\mathrm{Vf}}
$$

onde:

$$
\begin{array}{ll}
\text { ETp - Pe } & \text { - requerimento de água na área irrigada, } \mathrm{m}^{3} \\
\mathrm{Vf} & \text { - volume fornecido de água, } \mathrm{m}^{3}
\end{array}
$$

$\mathrm{O}$ valor de (ETp - Pe) para a área irrigada é determinado pela cultura, clima e intervalos entre aplicações de água, e foi calculado com o uso do modelo computacional CRIWAR (Bos et al., 1996), enquanto o volume fornecido (Vf) consiste do volume total entregue aos proprietários dos lotes irrigados.

Fornecimento médio de água: A definição, em termos de volume por hectare, dá uma indicação sobre o quanto de água está sendo utilizada por superfície de área irrigada. $O$ fornecimento médio de água (FMA) aos usuários do sistema será obtido em $\mathrm{m}^{3} \mathrm{ha}^{-1}$, conforme a expressão a seguir:

$$
\text { FMA }=\frac{\text { Vf }}{\text { Área irrigada }}
$$

\section{Indicadores financeiros}

O programa de transferência de perímetros públicos para administração privada é planejado para assegurar, às associações de usuários ou ao distrito de irrigação, os recursos financeiros para sua auto-suficiência (Johnson, 1997). Isto significa que o valor das tarifas de água geradas pelo distrito 
de irrigação, tem que ser suficiente para pagar os custos de operação, administração, manutenção do canal principal e da fonte de água do projeto. Os principais indicadores financeiros são:

Auto-suficiência financeira: A partir dos dados coletados de receitas geradas das tarifas de água (arrecadação) e dos gastos relativos à administração, operação, manutenção e energia do projeto (custo total), os índices de auto-suficiência financeira do distrito podem ser determinados, através da seguinte relação:

$$
\text { Auto }- \text { suficiência }=\frac{\text { Arrecadação }}{\text { Custo total }}
$$

Para minimizar os efeitos da inflação deve-se trabalhar com uma moeda estável.

Performance do pagamento da tarifa de água: De acordo com Bos (1997) a fração da dívida anual das tarifas a serem pagas ao distrito ou à associação de usuários é um importante indicador do nível de aceitação da distribuição de água para irrigação, como um serviço aos usuários. Ela é definida como:

Performance do pagamentoda tarifa de água $=\frac{\text { Arrecadação }}{\text { Faturamento }}$

A arrecadação representa o montante das tarifas pagas das contas vincendas e o faturamento é resultante da fatura das contas de água lançadas anualmente.

\section{Energia elétrica}

Considerando-se que a maioria dos perímetros irrigados captam água através do uso de estações de bombeamento e, portanto, os custos de energia elétrica incidem significativamente nos custos operacionais, inclui-se o estudo deste fator, a fim de se avaliar se as estações de bombeamento estão sendo operadas de forma adequada e com a racionalidade necessária. Para composição desta análise, utilizam-se os dados de volume fornecido, de área irrigada e as informações de consumo e demanda de energia no mês, obtidas através das contas de energia (Effertz et al., 1993).

De posse das contas lançadas mensalmente pela estação de bombeamento, os indicadores são obtidos de acordo com os seguintes índices:

consumo de energia em $\mathrm{kWh} \mathrm{ha}^{-1}$ irrigado

consumo de energia em $\mathrm{kWh} \mathrm{m}^{-3}$ fornecido

demanda de energia em $\mathrm{kW} \mathrm{ha}^{-1}$ irrigado

demanda de energia em $\mathrm{kW} \mathrm{m}^{-3}$ fornecido

O objetivo desta pesquisa é avaliar-se, de maneira global e quantitativa, dentro do contexto do Projeto RPIP, o Distrito de Irrigação Senador Nilo Coelho - DISNC, através da identificação de alguns indicadores visando à racionalização do uso dos recursos naturais e à sustentabilidade do sistema.

\section{MATERIAL E MÉTODOS}

A metodologia baseou-se em dados obtidos de relatórios e documentos de acompanhamento e avaliação da Companhia de Desenvolvimento do Vale do São Francisco - CODEVASF, e do Distrito de Irrigação Senador Nilo Coelho - DISNC, além de entrevistas com membros dessas instituições. Os dados coletados englobam séries temporais de parâmetros relacionados às superfícies irrigável e irrigada por cultura; volumes captados na estação de bombeamento principal (EBP) e fornecidos aos usuários; consumo e demanda de energia elétrica; faturas da tarifa de água e seu recebimento; custos financeiros do projeto (Distrito).

De posse das informações, fez-se uma análise da evolução dos indicadores de desempenho, enfocando-se os diferentes aspectos ligados ao seu comportamento durante o período de estudo.

Os dados meteorológicos foram obtidos da Estação Climática de Bebedouro da Empresa Brasileira de Pesquisa Agropecuária - EMBRAPA, situada em Petrolina, PE, cujo tipo climático é Bshw, ou seja, estépico de inverno seco, muito quente, segundo classificação de Köppen (EMBRAPA, 1981).

Com o uso do modelo CRIWAR 2.0 (Bos et al., 1996) determinaram-se a evapotranspiração de referência (ETo) pelo método de Penman-Monteith (Smith, 1991) e a precipitação efetiva (Pe) segundo metodologia proposta pelo Departamento de Agricultura dos Estados Unidos. Conhecido o valor da ETo, a evapotranspiração potencial das culturas pode ser estimada multiplicando-se esse valor pelos coeficientes de cultura (kc) obtidos por Doorenbos \& Pruitt (1977). Para cada ano, as onze principais culturas do DISNC foram consideradas tomando-se os dados relativos à área plantada e data de plantio.

O valor da tarifa de água foi calculado anualmente, através da adição de três parcelas: (a) da parcela de amortização dos investimentos públicos nas obras de infra-estrutura de irrigação $\left(\mathrm{k}_{1}\right)$ calculada em função da vida útil e dos hectares irrigáveis do projeto; (b) da parcela do custo fixo, correspondente ao rateio das despesas de administração, operação e manutenção, pela área total irrigável; e (c) da parcela do custo variável $\left(\mathrm{k}_{2}\right)$, correspondente ao valor das despesas de energia elétrica rateado pelo consumo de água no projeto.

\section{RESULTADOS E DISCUSSÃO}

\section{Sustentabilidade da área irrigada}

A sustentabilidade da área irrigada do DISNC apresentou valor de 0,29 em 1989, quando o projeto estava em fase de implementação e alcançou o máximo em 1995, seguido de uma pequena queda em 1996, mas voltando a se recuperar nos anos posteriores (Tabela 1).

Tabela 1. Sustentabilidade da área irrigada

\begin{tabular}{cccc}
\multirow{2}{*}{ Ano } & Área Irrigada & Área Irrigável & \multirow{2}{*}{ Sustentabilidade } \\
\cline { 2 - 3 } & \multicolumn{3}{c}{ (ha) } \\
\hline 1989 & 4.354 & 14.787 & 0,29 \\
1990 & 5.055 & 14.846 & 0,34 \\
1991 & 6.637 & 15.226 & 0,44 \\
1992 & 7.460 & 15.209 & 0,49 \\
1993 & 10.472 & 15.218 & 0,69 \\
1994 & 10.960 & 15.213 & 0,72 \\
1995 & 11.782 & 14.708 & 0,80 \\
1996 & 11.321 & 14.783 & 0,77 \\
1997 & 11.666 & 15.141 & 0,77 \\
1998 & 12.233 & 15.546 & 0,79 \\
\hline
\end{tabular}


O progressivo aumento na área irrigada, principalmente a partir de 1993, que coincide com a época de criação da Assistência Técnica e Extensão Rural - ATER, provavelmente seja fator de fundamental importância para o incremento na área irrigada, pois os pequenos produtores passaram a ter melhor acompanhamento do seu cultivo, além de ganho de conhecimento através de dias de campo, cursos etc. Souza (1999) afirma que a fruticultura colaborou para o avanço de novas áreas irrigadas no DISNC, na medida em que passou a ser uma alternativa econômica e viável para os produtores. Em 1990 havia 929,70 ha plantados com fruticultura, enquanto no ano de 1998 esta área atingiu 11.862,40 ha.

\section{Indicadores do balanço hídrico}

Os valores obtidos a partir do CRIWAR 2.0 e dos relatórios do DISNC, necessários para determinação dos indicadores fornecimento médio (FM) e razão global de consumo (RGC) são apresentados na Tabela 2. Observa-se redução no fornecimento médio de água aos usuários, de $16.850 \mathrm{~m}^{3} \mathrm{ha}^{-1}$, em 1990, a aproximadamente $13.600 \mathrm{~m}^{3} \mathrm{ha}^{-1}$, em 1998, com valor mínimo de $9.300 \mathrm{~m}^{3} \mathrm{ha}^{-1} \mathrm{em} \mathrm{1995.} \mathrm{Quanto} \mathrm{à} \mathrm{RGC,} \mathrm{verifica-se}$ que a mesma variou entre 0,33 a 0,74. Segundo Brito (1999) é provável que o valor satisfatório deste indicador se situe ao redor de 0,80 .

Os indicadores do balanço hídrico são influenciados pelo sistema de irrigação, pela eficiência de aplicação e pelo manejo adequado da irrigação. $\mathrm{O}$ aumento na RGC do projeto, principalmente a partir de 1992, coincide com a criação da Assistência Técnica do Distrito de Irrigação. Vale salientar que, durante este período, houve aumento da área irrigada por sistemas de irrigação localizada.

No Distrito Coello (Colômbia), houve redução no fornecimento médio anual de água, de aproximadamente $11.000 \mathrm{~m}^{3} \mathrm{ha}^{-1}, \mathrm{em} \mathrm{1982,}$ para $9.700 \mathrm{~m}^{3} \mathrm{ha}^{-1}$, em 1991. Esta tendência foi influenciada por duas mudanças básicas. A primeira foi a expansão na área total irrigada, de aproximadamente 21.000 ha em 1977 para a faixa compreendida entre 27.000 e 37.000 ha, no final da década de 1980 e início da década de 1990, respectivamente. A segunda foi a mudança do monocultivo do arroz para outras culturas, principalmente algodão, sorgo e soja (Vermillion \& GarcezRestrepo, 1996).

Analisando-se os resultados encontrados nas Tabelas 1 e 2, verifica-se a interdependência entre os indicadores, pois se observa que, ao longo do tempo, os indicadores de sustentabilidade da área irrigada e RGC apresentaram melhores desempenhos. Além dos fatores climáticos e de sustentabilidade da área irrigada, os quais influem diretamente o volume de água demandado, as mudanças dos sistemas de aspersão convencional para sistemas de irrigação localizada (microaspersão e gotejamento) e a melhoria do manejo da irrigação, possivelmente têm contribuído para o aumento da eficiência no uso da água.

\section{Indicadores financeiros}

Na Tabela 3 são apresentados os parâmetros para determinação da auto-suficiência financeira do DISNC. Os valores de arrecadação, custo total e fatura da tarifa, foram obtidos através da média mensal para o ano analisado. Como não foi possível separar do conjunto total de custos os relacionados unicamente aos de operação e manutenção, utilizou-se o custo total do perímetro, considerando-se os custos fixos e variáveis.

Observa-se que a auto-suficiência do Distrito tendeu a crescer nos cinco primeiros anos, porém decresceu nos últimos dois anos, fato este que pode ser explicado pela ocorrência dos fortes ventos no final de 1997 e começo de 1998, os quais provocaram queda considerável do bananal, com reflexos diretos sobre a receita, principalmente dos pequenos produtores (colonos). De acordo com dados da ATER, foram atingidos 810 produtores, com perdas em 2.596 ha, alcançando um prejuízo estimado sobre a receita em $\mathrm{R} \$ 13,0$ milhões.

No Projeto de Irrigação Samaca, na Colômbia, Fraiture \& Garcez-Restrepo (1997) fizeram uma análise temporal com o uso de indicadores de desempenho e constataram que no período entre 1986 e 1990, a auto-suficiência média foi de 35\%. Com a transferência do manejo de irrigação e aumento significativo da tarifa de água, esta situação mudou drasticamente.

Tabela 2. Fornecimento médio de água e razão global de consumo (RGC) entre os anos de 1990 e 1998

\begin{tabular}{|c|c|c|c|c|c|c|}
\hline Ano & $\begin{array}{l}\text { Área Irrigada } \\
\text { (ha) }\end{array}$ & ETp & $\frac{\mathrm{Pe}}{\left(10^{3} \mathrm{~m}^{3}\right)}$ & $\mathrm{Vf}$ & $\begin{array}{l}\text { Fornecimento Médio } \\
\qquad\left(10^{3} \mathrm{~m}^{3} \mathrm{ha}^{-1}\right)\end{array}$ & RGC \\
\hline 1990 & 5.055 & 37.548 & 9.092 & 85.164 & 16,85 & 0,33 \\
\hline 1991 & 6.637 & 58.060 & 20.492 & 91.332 & 13,76 & 0,43 \\
\hline 1992 & 7.460 & 63.743 & 21.714 & 89.106 & 11,94 & 0,47 \\
\hline 1993 & 10.472 & 103.884 & 11.754 & 149.637 & 14,29 & 0,62 \\
\hline 1994 & 10.960 & 123.152 & 34.669 & 128.664 & 11,74 & 0,69 \\
\hline 1995 & 11.782 & 117.685 & 43.975 & 109.568 & 9,30 & 0,67 \\
\hline 1996 & 11.321 & 123.198 & 35.095 & 129.916 & 11,48 & 0,68 \\
\hline 1997 & 11.666 & 117.554 & 32.721 & 114.212 & 9,79 & 0,74 \\
\hline 1998 & 12.233 & 135.263 & 30.145 & 166.398 & 13,60 & 0,63 \\
\hline
\end{tabular}

Tabela 3. Auto-suficiência financeira do DISNC

\begin{tabular}{|c|c|c|c|c|c|}
\hline \multirow{2}{*}{ Ano } & Arrecadação & Faturamento & \multirow{2}{*}{$\begin{array}{l}\text { Performance de } \\
\text { Pagamento }\end{array}$} & \multirow{2}{*}{$\begin{array}{l}\text { Custo Total } \\
\text { (US\$) }\end{array}$} & \multirow{2}{*}{ Auto-suficiência } \\
\hline & & & & & \\
\hline 1992 & 126.767 & 145.281 & 0,87 & 133.004 & 0,95 \\
\hline 1993 & 180.532 & 194.046 & 0,93 & 163.308 & 1,11 \\
\hline 1994 & 235.930 & 299.997 & 0,79 & 240.459 & 0,98 \\
\hline 1995 & 386.690 & 369.210 & 1,05 & 273.200 & 1,42 \\
\hline 1996 & 406.585 & 388.280 & 1,05 & 288.886 & 1,41 \\
\hline 1997 & 325.289 & 319.759 & 1,02 & 261.084 & 1,25 \\
\hline 1998 & 279.838 & 304.903 & 0,92 & 306.386 & 0,91 \\
\hline
\end{tabular}


Tabela 4. Consumo e demanda de energia por volume fornecido e área irrigada entre os anos de 1989 - 1998 no DISNC

\begin{tabular}{ccccccccccc}
\hline Descrição & 1989 & 1990 & 1991 & 1992 & 1993 & 1994 & 1995 & 1996 & 1997 & 1998 \\
\hline $\begin{array}{c}\text { Consumo } \\
(1000 \mathrm{kWh})\end{array}$ & 36.016 & 34.529 & 32.174 & 33.741 & 53.204 & 42.357 & 38.765 & 41.690 & 35.284 & 55.539 \\
$\begin{array}{c}\text { Consumo } \\
\left(\mathrm{kWh} \mathrm{m}^{-3}\right)\end{array}$ & 0,50 & 0,41 & 0,35 & 0,38 & 0,36 & 0,33 & 0,35 & 0,32 & 0,31 & 0,33 \\
$\begin{array}{c}\text { Consumo } \\
\left(\mathrm{kWh} \mathrm{ha}^{-1}\right)\end{array}$ & 689,26 & 569,24 & 403,98 & 376,91 & 423,39 & 322,05 & 274,19 & 306,88 & 252,05 & 378,33 \\
$\begin{array}{c}\text { Demanda } \\
\left(1000 \mathrm{~kW}^{2}\right)\end{array}$ & 185 & 169 & 212 & 164 & 270 & 193 & 215 & 211 & 201 & 194 \\
$\begin{array}{c}\text { Demanda } \\
\left(10^{-3} \mathrm{~kW} \mathrm{~m}^{-3}\right)\end{array}$ & 2,57 & 1,98 & 2,32 & 1,84 & 1,80 & 1,50 & 1,96 & 1,62 & 1,76 & 1,17 \\
$\begin{array}{c}\text { Demanda } \\
\left(\mathrm{kW} \mathrm{ha}^{-1}\right)\end{array}$ & 3,54 & 2,79 & 2,66 & 1,83 & 2,15 & 1,47 & 1,52 & 1,55 & 1,44 & 1,32 \\
\hline
\end{tabular}

A auto-suficiência subiu de 50\% em 1991, para 109\% no ano seguinte. Nesse momento o governo reduziu os subsídios a zero e todos os custos para manter e operar o sistema foram cobertos pelos próprios usuários.

$\mathrm{O}$ aumento na arrecadação está relacionada às medidas tomadas pelo DISNC, principalmente com a suspensão do fornecimento de água aos lotes inadimplentes, de acordo com as seguintes condições:

- até 1993: corte de água para débitos acima de 12 meses

- de 1994 a setembro de 1996: corte para débitos acima de 6 meses

- outubro de 1996: corte de água para débitos acima de 3 meses, somente após esgotadas várias negociações.

A partir da realização dessas medidas, houve aumento significativo no pagamento de contas em atraso, o qual proporcionou valores de arrecadação superiores ao faturado nos anos de 1995, 1996 e 1997, ressaltando-se que o aumento da tarifa e as medidas de corte de água tomadas a partir de 1993, possivelmente foram os responsáveis pelo aumento do desempenho financeiro.

Johnson (1997) verificou que os aumentos da tarifa de água realizados no México foram associados ao programa de transferência do manejo de irrigação do órgão público para associações de usuários, que proporcionou um aumento na auto-suficiência financeira, de 37\% em 1991 a 80\% em 1994. Devido à exigência do pagamento das tarifas, antes do fornecimento de água aos produtores, este país tem alcançado níveis de performance de pagamento ao redor de $100 \%$.

Vale salientar que a performance de pagamento não reflete apenas o desempenho financeiro mas, também, e em parte, o nível de satisfação do usuário com respeito à atuação do Distrito de Irrigação.

\section{Consumo e demanda de energia}

Observa-se, na Tabela 4, que o consumo de energia por volume fornecido e por área irrigada, apresentou decréscimo durante os anos de 1989 a 1998 , variando de 0,50 a aproximadamente $0,30 \mathrm{KW} \mathrm{h}^{-1} \mathrm{~m}^{-3} \mathrm{e}$ de $689,26 \mathrm{a} 378,33 \mathrm{~kW} \mathrm{~h}^{-1} \mathrm{ha}^{-1}$.

$\mathrm{O}$ aumento da sustentabilidade da área irrigada representa um avanço na ocupação do projeto, propiciando maior uso do sistema de irrigação, o qual foi projetado para irrigar uma área de 15.000 ha. Desta forma, as estações de bombeamento aumentam sua eficiência, pois ficam trabalhando dentro da sua capacidade de trabalho e o retorno econômico, através do fluxo de caixa gerado pelas tarifas de água, é incrementado.
A demanda de potência por volume fornecido e por hectare irrigado, que representa a potência requerida, ou necessária para operar a estação de bombeamento, também apresentou o mesmo comportamento, cujos valores variaram de 2,57 a $1,17 \times 10^{-3} \mathrm{KW} \mathrm{m}^{-3}$ e de $3,54 \mathrm{a} 1,32 \mathrm{~kW} \mathrm{ha}^{-1}$, respectivamente, demonstrando, portanto, que as estações apresentaram, ao longo do tempo, um uso mais racional de energia.

Por se constituir na maior parcela do custo total do projeto, a redução desses números constitui uma redução no custo variável, que incide sobre a tarifa de água. Como a água é o insumo mais importante para a agricultura irrigada, recomenda-se trabalhar com os indicadores em função do volume fornecido.

Embora o aumento da área irrigada e, conseqüentemente, do volume fornecido, tenha sido responsável por esta economia de energia, destaca-se que alguns fatores, segundo informações fornecidas pelo DISNC, também contribuíram de maneira decisiva, tais como: (a) instalação gradual de bombas automáticas, com conseqüente operação contínua e custo energético mais baixo, entre 21:00 e 5:00 h; (b) melhoria no manejo de irrigação; (c) mudança no tipo de sistema de irrigação nos lotes, de aspersão para sistemas localizados; e (d) melhor operacionalização através do treinamento de canaleiros e inspetores de irrigação, evitando-se perdas por transbordamento em reservatórios e canais.

\section{CONCLUSÕES}

Com base nos resultados encontrados, foi possível tirar-se algumas conclusões gerais sobre o desempenho do Distrito de Irrigação Senador Nilo Coelho, que são:

1. Os resultados encontrados demonstraram que não houve nenhuma interrupção no processo de ocupação das áreas irrigáveis, indicando que, do ponto de vista macro-gerencial, a implementação do projeto tem ocorrido com sucesso.

2. A razão global de consumo apresentou tendência de alta ao longo do tempo, com intervalos entre 0,33 e 0,74 .

3. No aspecto financeiro, o Distrito apresentou tendência de alta no índice de auto-suficiência, até o ano de 1996 porém, com o impacto da queda de extensas áreas cultivadas com banana, houve expressiva redução deste indicador nos anos de 1997 e 1998.

4. A atuação mais firme por parte do Distrito, através do corte de água, proporcionou elevação nos índices de performance de pagamento dos usuários do sistema.

5. Os indicadores de consumo e demanda de energia apontam para uma racionalização melhor no uso da energia elétrica, durante o período analisado.

6. Existe interdependência entre os indicadores de desempenho. 


\section{LITERATURA CITADA}

Bos, M.G. Performance indicators for irrigation and drainage. Irrigation and Drainage Systems, Dordrecht, v.11, n.2, p.119-137, 1997.

Bos, M.G.; Vos, J.; Feedders, R.A. CRIWAR 2.0. A simulation model on crop irrigation water requirements. Wageningen: International Institute for Land Reclamation and Improvement (ILRI), 1996. 117p. ILRI. Publication, 46

Brito, R.A.L. Avaliação do desempenho de um perímetro irrigado - Proposta para um modelo conceitual. In: Congresso Nacional de Irrigação e Drenagem, 7, 1986, Brasília. Anais... Brasília: Associação Brasileira de Irrigação e Drenagem, 1986. p.749-77Brito, R.A.L. Avaliação do desempenho da irrigação no Brasil (RPIP - Brasil). Sete Lagoas, EMBRAPA/SRH/IICA/ FAPED/DHV. 1999. 51p. Relatório n.3

Brito, R.A.L.; Soares, J.M.; Cavalcanti, E.B.; Bos, M.G. Irrigation performance assessment for Nilo Coelho Scheme in Northeastern Brazil: A preliminary analysis. In: Afro-Asian Regional Conference, 10, 1998, Proceedings... Bali: ICID, 1998. v.II-A, A13.1-7.

Doorenbos, J.; Pruitt, W.O. Necessidad de agua de los cultivos. Roma: FAO, 1977. 194p. Estudio FAO. Riego y Drenage, 24

Effertz, R.; Olson, D.C.; Vissia, R.; Arrunategui, H. Operação e manutenção de projetos de irrigação. In: Manual de irrigação. Brasília: Secretaria da Irrigação, v.4, 1993.490p.

EMBRAPA - Empresa Brasileira de Pesquisa Agropecuária. Serviço de produção de sementes básicas: Implantação do complexo da produção de sementes de Petrolina, PE. Brasília: EMBRAPA, 1981.75p.
Fraiture, C.; Garcéz-Restrepo, C. Assessing trends and changes in irrigation performance. The case of Samaca Irrigation Scheme, Colombia. International Seminar: Research Program on Irrigation Performance (RPIP). Mendoza, Argentina. 1997. $19 \mathrm{p}$.

Johnson III, S.H. Management transfer in Mexico: A strategy to achieve irrigation district sustainability. Colombo, Sri Lanka: International Irrigation Management Institute (IIMI). 1997. 31p. Research Report n.16

Merrey, D.J. Institutional design principles for accountability in large irrigation systems. Colombo, Sri Lanka: International Irrigation Management Institute (IIMI). 1996. Research Report n.8

Molden, D.J.; Sakthivadivel, R.; Perry, C.J.; Fraiture, C.; Kloezen, W.H. Indicators for comparing performance of irrigated agricultural systems. Colombo, Sri Lanka: International Irrigation Management Institute (IIMI), 1998. 26p. Research Report, n.20

Smith, M. Report on the expert consultation on revision of FAO methodologies for crop water requirements. Rome: FAO. 1991.45p.

Souza, G.H.F. Indicadores de desempenho para avaliação de perímetros irrigados: $\mathrm{O}$ caso do distrito de irrigação Senador Nilo Coelho. Campina Grande: UFPB, 1999. 59p. Dissertação Mestrado

Vermillion, D.L.; Garcéz-Restrepo, C. Results of management turnover in two irrigation districts in Colombia. Colombo, Sri Lanka: International Irrigation Management Institute (IIMI), 1996. 32p. Research Report n.4 